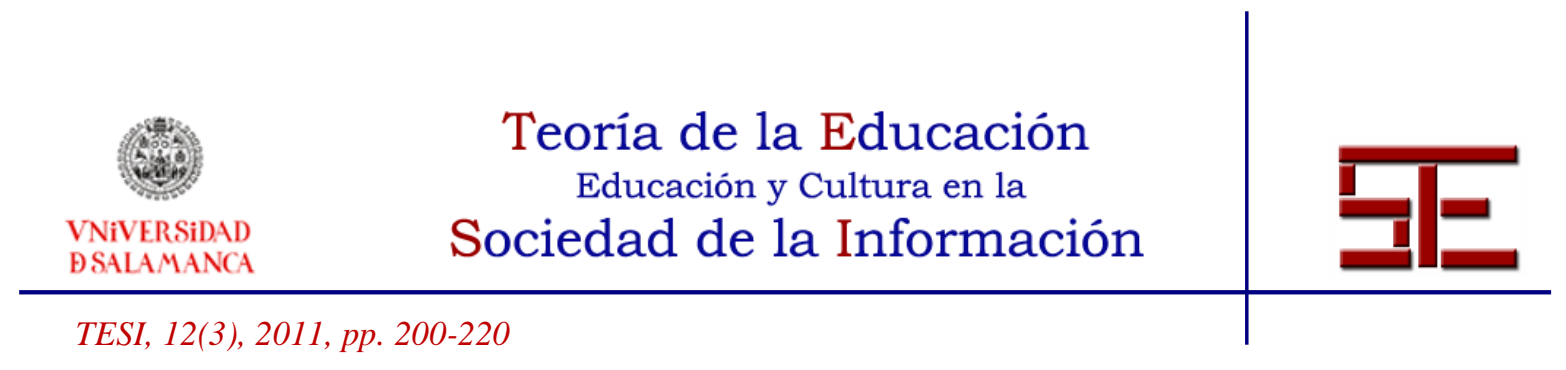

\title{
ACTITUDES ANTE EL APRENDIZAJE EN ESTUDIANTES UNIVERSITARIOS EXCELENTES Y EN ESTUDIANTES MEDIOS
}

Resumen: Pretendemos analizar lo que hacen los alumnos con mejor nota de entrada en la universidad que pueda explicar su rendimiento, de cara a precisar modelos eficaces de funcionamiento que se puedan generalizar y enseñar, en la medida de lo posible. Usando el cuestionario CEVAPU (Cuestionario de evaluación de las actitudes ante el aprendizaje de los estudiantes universitarios), evaluamos las actitudes ante el aprendizaje de un grupo de alumnos excelentes, seleccionados de 11 titulaciones de 9 centros de la Universidad Politécnica de Valencia, y comparamos los resultados con un grupo de $\mathrm{N}$ similar de alumnos medios de los mismos centros. Encontramos que los alumnos excelentes desarrollaban mejores actitudes que los alumnos medios. Hallamos también que las actitudes ante el aprendizaje tenían influencia en el rendimiento académico. Los datos de que disponemos permiten afirmar que los profesores pueden potenciar las actitudes ante el aprendizaje usando metodologías de enseñanza y evaluación pertinentes.

Palabras clave: Actitudes ante el aprendizaje; estudiantes universitarios; estudiantes excelentes; rendimiento académico.

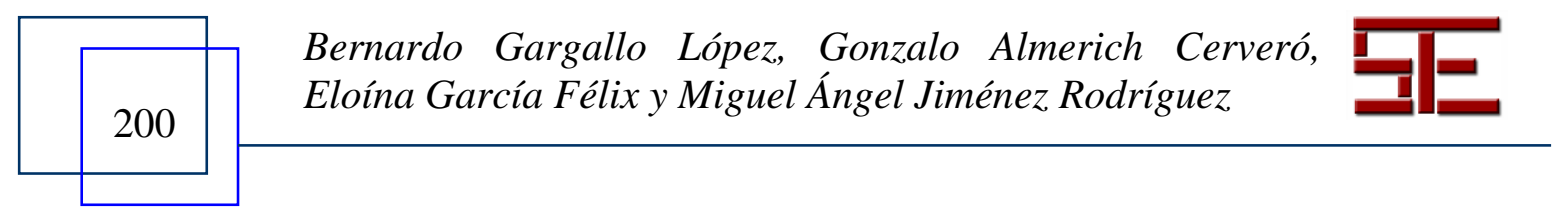




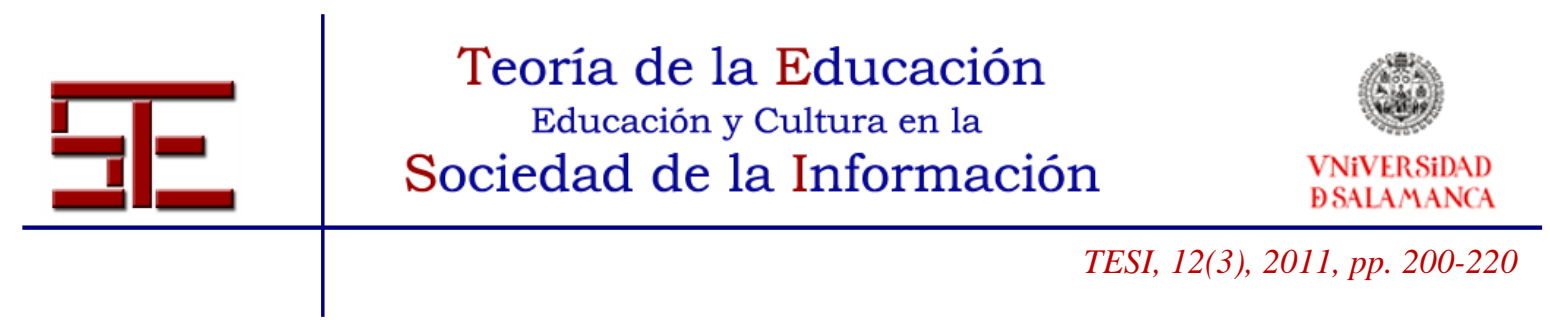

\title{
LEARNING ATTITUDES IN EXCELLENT AND AVERAGE UNIVERSITY STUDENTS
}

\begin{abstract}
We seek to analyse how the best students on entry to University work in order to transfer it to the rest of the University community. We used the AUSLQ questionnaire (Attitudes of University Students toward Learning Questionnaire) to assess the attitudes of a sample of 148 excellent students selected from 11 degrees from 9 centers of the Polytechnic University of Valencia, and we compared the results with those of a sample of 133 average students of the same centers. We found that excellent students developed better attitudes than average students. We also found that learning attitudes had an influence on the academic achievement. The available data allow us to affirm that the professors can enhance the deep approach by using adequate teaching and assessment methodologies.
\end{abstract}

Keywords: Learning attitudes; university students; excellent students; academic achievement.

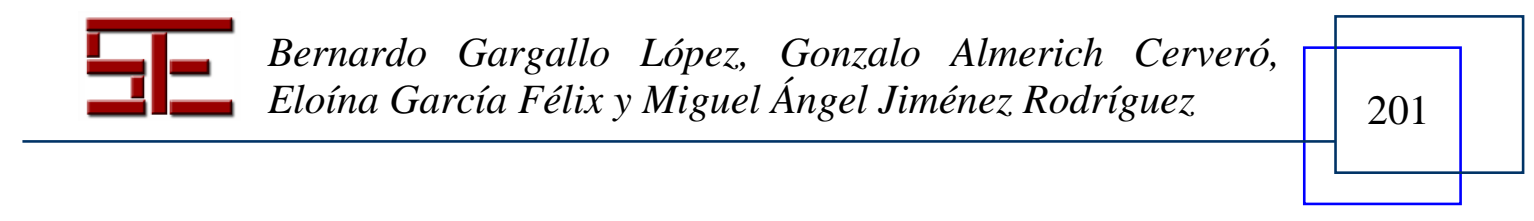




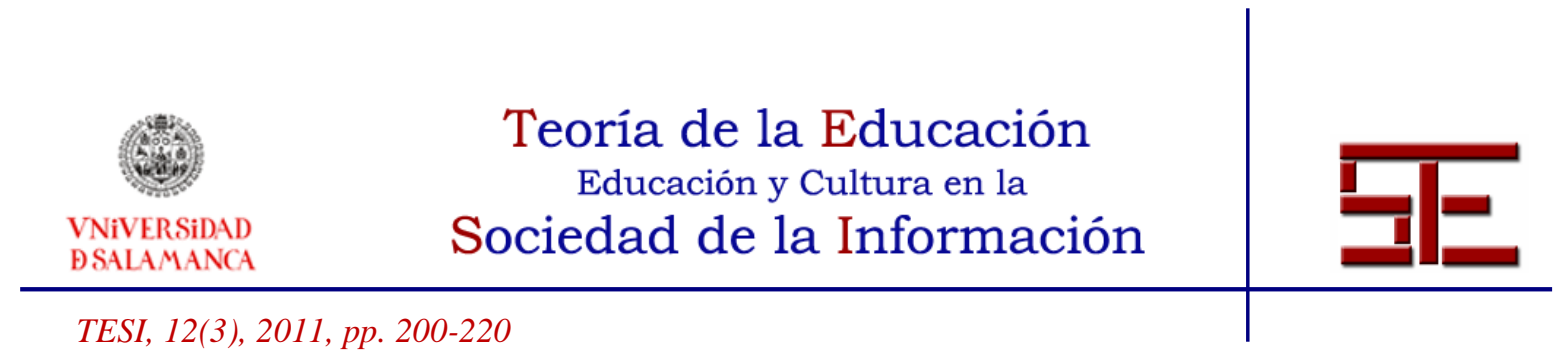

\title{
ACTITUDES ANTE EL APRENDIZAJE EN ESTUDIANTES UNIVERSITARIOS EXCELENTES Y EN ESTUDIANTES MEDIOS
}

Fecha de recepción: 21/07/2011; fecha de aceptación: 15/09/2011; fecha de publicación: 30/11/2011

\author{
Bernardo Gargallo López \\ bernardo.gargallo@uv.es \\ Universidad de Valencia \\ Gonzalo Almerich Cerveró \\ gonzálo.almerich@uv.es \\ Universidad de Valencia
}

Eloína García Félix

algarcia@upv.es

Universidad Politécnica de Valencia

Miguel Ángel Jiménez Rodríguez

mangel.jimenez@ucv.es

Universidad Católica de Valencia "San Vicente Mártir"

\section{1.- INTRODUCCIÓN}

Los resultados que aquí presentamos se inscriben en el contexto de una investigación de tres años ${ }^{1}$, que pretende analizar lo que hacen los alumnos con mejor nota de entrada en la universidad que pueda explicar su rendimiento, de cara a precisar modelos eficaces de funcionamiento que se puedan generalizar y enseñar, en la medida de lo posible. Para ello se está analizando el modo de aprender varios grupos de alumnos de 1er curso de diferentes titulaciones de la Universidad Politécnica de Valencia con las mejores notas de acceso a la universidad. Sus resultados se compararán con alumnos de calificaciones

\footnotetext{
${ }^{1}$ Se trata de "La excelencia en los estudiantes universitarios desde un enfoque longitudinal: Análisis de factores incidentes y diseño de un modelo de intervención", investigación aprobada por el Ministerio de Ciencia e Innovación de España dentro del Programa Nacional de Proyectos de Investigación Fundamental, en el marco del V Plan de Investigación Científica, Desarrollo e Innovación Tecnológica convocatoria de 2009 (2010-2012) (Financiación Plan E, PGE) (código EDU2009/08518).
}

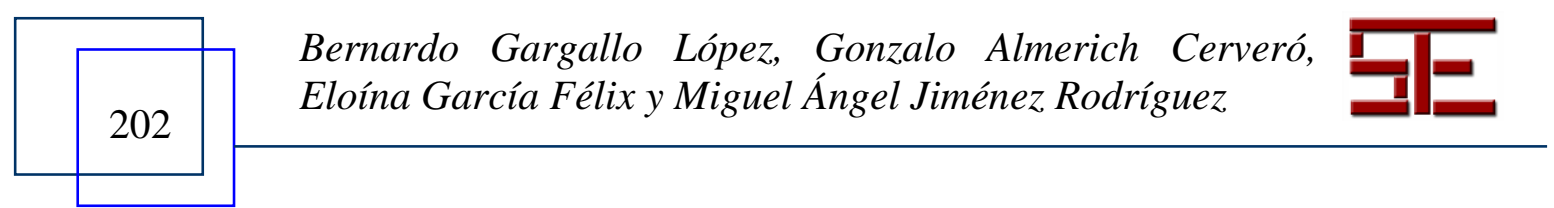




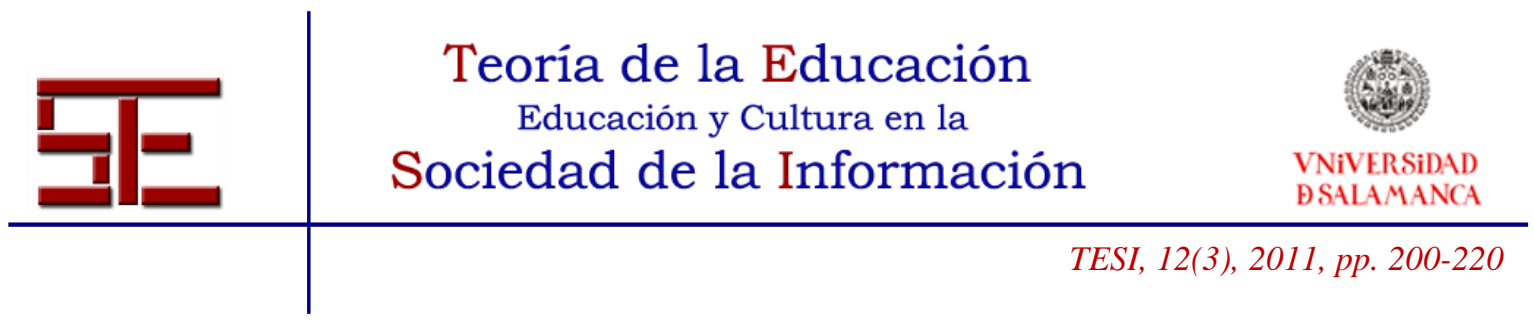

medias de sus mismas titulaciones. Con ese objetivo estamos valorando sus estrategias de aprendizaje, enfoques, estilos, actitudes ante el aprendizaje y otras variables relevantes (autoconcepto, C.I., percepción que estos alumnos tienen de sus profesores y de sus clases, integración en la universidad, valoraciones que hacen de ellos sus profesores, etc.). En esta investigación vamos a llevar a cabo un seguimiento de su evolución durante sus dos primeros cursos de permanencia en la universidad, con varias medidas para recoger datos a lo largo del periodo de la investigación.

En este trabajo nos centramos en los resultados referidos a las actitudes ante el aprendizaje de los estudiantes. Queremos precisar, a partir de una primera toma de datos realizada en la investigación, las posibles diferencias existentes en actitudes entre los dos colectivos de alumnos (excelentes y medios). Así mismo, la influencia que éstas tienen sobre el rendimiento académico. Éstos son los objetivos que pretendemos valorar y a ello se refieren los resultados que presentamos en este trabajo. Tenemos la convicción de que los estudiantes excelentes desarrollan mejores actitudes ante el aprendizaje que los estudiantes medios, y también que las actitudes ante el aprendizaje influyen en el rendimiento académico del estudiante, de modo que un elemento importante del perfil del estudiante excelente debe ser el desarrollo de buenas actitudes ante el aprendizaje.

\section{2.- FUNDAMENTACIÓN}

En la presente investigación partimos de una concepción de la actitud como tendencia o predisposición aprendida y relativamente duradera a evaluar de determinado modo a un objeto, persona, grupo, suceso o situación, a partir de las creencias disponibles en torno a los mismos. Tal tendencia o predisposición conduce a actuar, de modo favorable o desfavorable, hacia ese objeto, persona, grupo, suceso o situación, de manera consecuente con dicha evaluación. Las actitudes son predisposiciones estables a valorar y actuar que se basan en una organización relativamente duradera de creencias en torno a la realidad que predispone a actuar de determinada forma (Escámez, García, Pérez y Llopis, 2007; Escámez y Ortega, 1986; García y Sales, 1997; Rokeach, 1970 y 1979; Wander Zanden, 1989).

Las actitudes tienen un carácter multidimensional que integra diversos componentes: cognitivo, afectivo-evaluativo y conductual, aunque para la mayoría de los autores el componente afectivo-evaluativo se considera como el elemento más esencial o específico de la actitud.

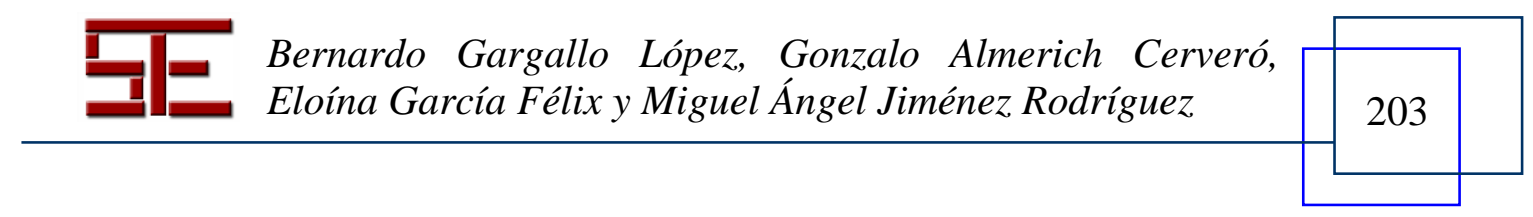




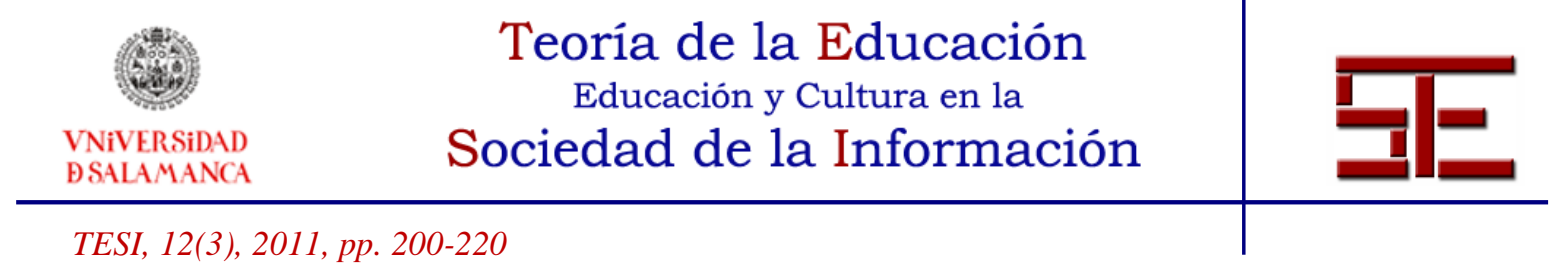

Aunque las variables que condicionan el rendimiento académico de los estudiantes universitarios son muy numerosas y constituyen una intrincada red en la que resulta harto complejo ponderar la influencia específica de cada una, partimos de la idea de que las actitudes que mantienen los estudiantes hacia el aprendizaje son una de las variables fundamentales que influye en los resultados escolares. Se trata, pues, de un tema sumamente relevante en el ámbito educativo.

La preocupación por las actitudes hacia el estudio de los alumnos viene de antiguo. No obstante, en nuestro tiempo esta cuestión adquiere un renovado interés, en gran medida por la extensión de la educación, así como por las altas tasas de fracaso escolar. De hecho, diversas investigaciones se orientan a precisar los procesos implicados en el aprendizaje y a valorar en qué grado influyen las actitudes de los estudiantes hacia el aprendizaje y el estudio en el rendimiento académico. Hay algunos trabajos que reflejan la relación existente entre actitudes y rendimiento, generalmente de estudiantes no universitarios. Así el de Quiles (1993) o el de Ramírez (2005), que comprueban la incidencia de las actitudes en el rendimiento de los estudiantes de primaria y el de Akey (2006) en estudiantes de secundaria. No hay muchos trabajos fuera de nuestro país que analicen la influencia de las actitudes en el rendimiento de estudiantes universitarios (Goolsby, 1988; House y Prion, 1998). Tampoco en España: los estudios de Herrero, Nieto, Rodríguez y Sánchez (1999) y de Gargallo, Pérez, Serra, Sánchez y Ros (2007) son de los pocos que analizan la incidencia de las actitudes, en este caso con población universitaria, concluyendo que es una variable con influencia en el rendimiento.

Llama la atención la escasez de prospecciones de este tipo en universitarios, acaso porque se supone que los estudiantes que ingresan en la universidad poseen unas actitudes muy favorables hacia el estudio. Sin embargo, la experiencia nos demuestra que un número significativo de alumnos de enseñanza superior obtienen malos resultados. En efecto, no todos los estudiantes hacen frente con éxito a los nuevos desafíos que la universidad plantea: aumento de la exigencia, necesidad creciente de organización del trabajo académico, mayor dedicación al estudio, autonomía, etcétera.

Por otra parte, el tema no ha sido abordado en el contexto a que hace referencia nuestra investigación, el análisis del perfil del estudiante excelente centrado en los primeros cursos de la universidad.

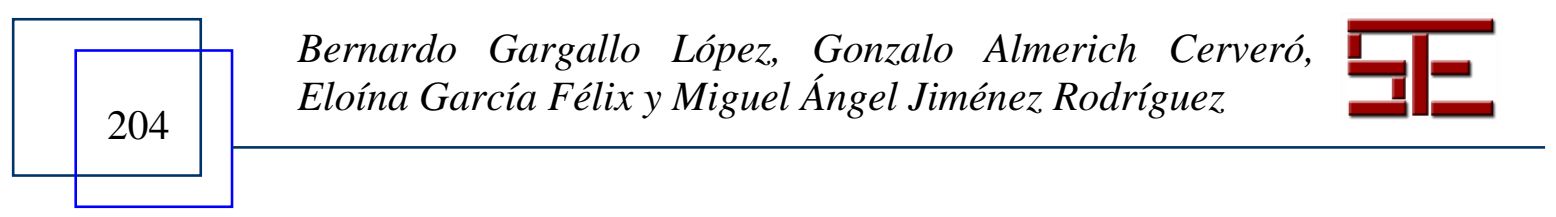




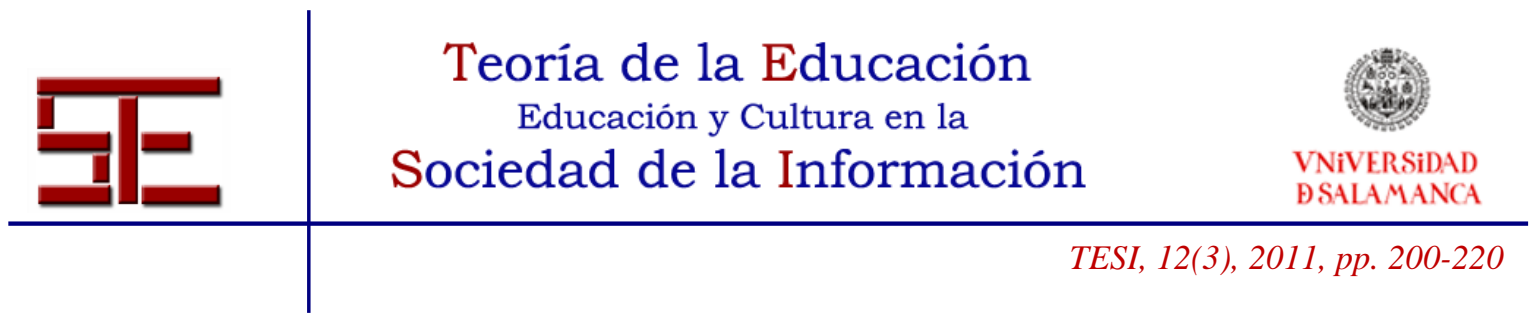

Es éste, el de los estudiantes excelentes, un tema poco abordado en la investigación. Hay diversos estudios realizados sobre el funcionamiento de los estudiantes en su primer año universitario: analizando las variables predictoras de un buen ajuste (Pritchard, Wilson y Yamnitz, 2007), el impacto de la estructura familiar (Deronck, 2007) o el apoyo social y el estrés académico (Rayle y Chung, 2007). También encontramos estudios que analizan los factores que influyen en el rendimiento académico, de estudiantes nativos (Fore, 1998) o de estudiantes cuyos padres no fueron a la universidad (Strayhorn, 2006). Existen también investigaciones sobre rendimiento a lo largo de los estudios (De Miguel y Arias, 1999; Meléndez, 2007).

Pero no hemos podido constatar la existencia de datos de investigaciones sobre modos de actuación de estudiantes excelentes (que entendemos como los alumnos que obtienen las mejores notas) que analicen las diversas variables relevantes involucradas en su aprendizaje para determinar cuál es su modo de funcionar (habilidades y estrategias, motivación, autoconcepto, actitudes, expectativas, integración en la universidad, etc.), frente a los que no lo son.

Entendemos, pues, que nuestra aportación puede enriquecer el conocimiento pedagógico y dar pie para elaborar propuestas de acción educativa.

\section{3.- MÉTODO}

\section{1.- Diseño}

La investigación se estructura con un diseño cuasiexperimental de grupo de control no equivalente, complementado con metodología de tipo descriptivo-exploratorio (estudio de encuesta) y correlacional.

\section{2.- Participantes}

Seleccionamos 11 grupos de alumnos pertenecientes a 11 titulaciones de 9 centros de la Universidad Politécnica de Valencia. Las 11 titulaciones eran: Ingeniero Técnico Industrial, Ingeniero Técnico en Diseño Industrial, Ingeniero Técnico en Informática, Ingeniero Técnico en Obras Públicas, Arquitecto Técnico, Arquitecto, Ingeniero de Telecomunicaciones, Ingeniero de Caminos, Canales y Puertos, Ingeniero Industrial, Licenciado en Bellas Artes, y Licenciado en Biotecnología. Los 9 centros eran: la Escuela Técnica Superior de Ingeniería del Diseño, la Escuela Técnica Superior de

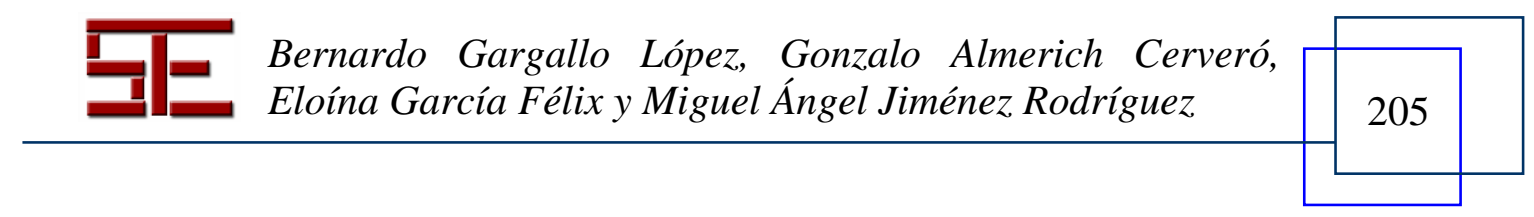




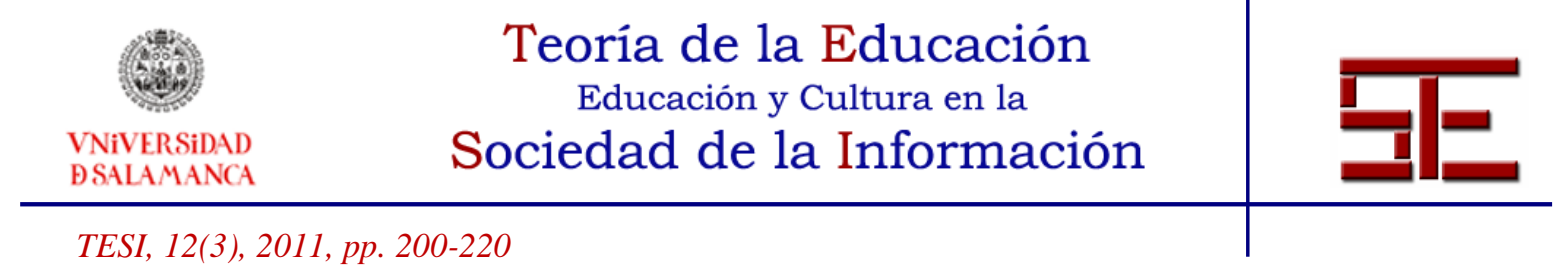

Informática, la Escuela Técnica Superior de Ingenieros de Caminos, Canales y Puertos, la Escuela Técnica Superior de Ingenieros Industriales, la Escuela Técnica Superior de Ingenieros Agrónomos, la Facultad de Bellas Artes, la Escuela Técnica Superior de Telecomunicaciones, la Escuela Técnica Superior de Gestión de la Edificación y la Escuela Técnica Superior de Arquitectura.

La muestra estaba integrada, en el primer pase del cuestionario de enfoques de aprendizaje, por 269 alumnos, 146 excelentes y 123 medios. La muestra mínima proyectada (que deseábamos mantener durante los dos años de la investigación) era de 10 alumnos excelentes y otros 10 medios por cada uno de los grupos, lo que daba un total de 220 alumnos. Sin embargo, dado que existían previsiones más que razonables de mortalidad experimental a lo largo de los dos años de la investigación, la muestra seleccionada inicialmente fue ligeramente mayor, acercándose a los 300 sujetos, de los que obtuvimos respuesta de 269 al cuestionario de enfoques de aprendizaje en el primer pase de instrumentos.

De cada una de las titulaciones fueron seleccionados como sujetos excelentes los que habían obtenido las notas más altas de la titulación en la Prueba de Acceso a la Universidad. Como estudiantes medios fueron seleccionados los que se ubicaban alrededor de la mediana de la titulación, por encima y por debajo de la misma de forma equilibrada. La selección se realizó en septiembre, al inicio del curso.

\section{3.- Instrumento de medida}

Utilizamos el cuestionario CEVAPU (Cuestionario para la Evaluación de las Actitudes hacia el Aprendizaje en Estudiantes Universitarios), un instrumento diseñado y validado por el equipo investigador (Gargallo, Pérez, Fernández y Jiménez, 2007) en una investigación anterior ${ }^{2}$ para valorar las actitudes hacia el aprendizaje de los estudiantes universitarios al no encontrar ninguno adecuado para cubrir los objetivos de la investigación.

\footnotetext{
${ }^{2}$ Se trata de la investigación "Estrategias de enseñanza y estrategias de aprendizaje en la universidad. Análisis de la incidencia de variables fundamentales en los modos en que los alumnos afrontan el aprendizaje" (código SEC2003-06787/PSCE), aprobada por el Ministerio de Ciencia y Tecnología de España por medio de convocatoria pública de tipo competitivo, y financiada por el Ministerio de Ciencia y Tecnología y por el FEDER (Fondo Europeo de Desarrollo Regional).
}

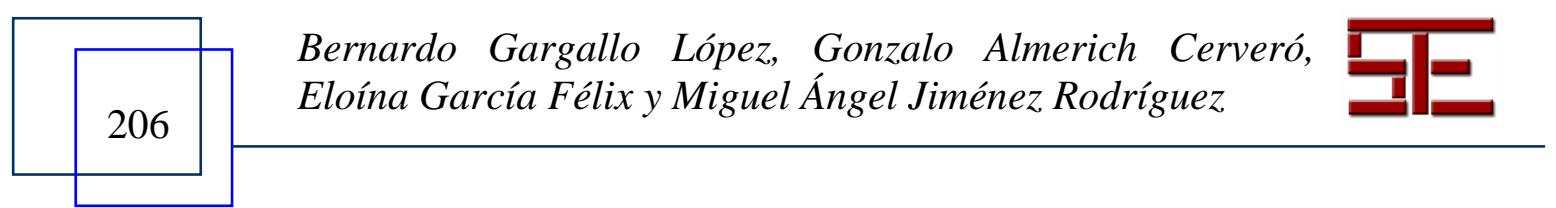




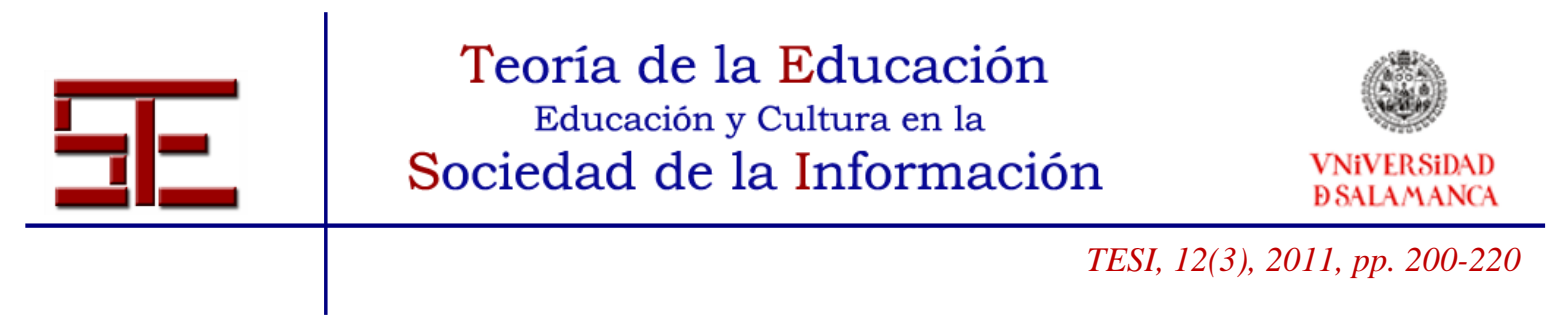

El cuestionario, constituido por once ítems que se agrupan en tres dimensiones, adopta el formato de las escalas tipo Likert, con cinco opciones de respuesta, que van desde "muy en desacuerdo" hasta "muy de acuerdo". Es un cuestionario elaborado a partir de del modelo de la teoría de la acción razonada de Fishbein y Ajzen (1980), al entender que constituye la teoría más completa y la única general que explica los procesos de formación y cambio de actitudes. Esta teoría engloba creencias, normas subjetivas, actitudes, intención y conducta.

La estructura factorial del cuestionario resultante se recoge en la Tabla 1. El instrumento tiene un alto nivel de consistencia interna. El coeficiente alfa de Cronbach para todo el cuestionario es de .701 y para cada una de las dimensiones o factores se recoge en la Tabla 1.

\begin{tabular}{|l|c|c|}
\hline \multicolumn{1}{|c|}{ Factores/dimensiones } & Fiabilidad & Número de ítems \\
\hline $\begin{array}{l}\text { Factor 1.- Actitud positiva hacia el aprendizaje } \\
\text { profundo, crítico, con comprensión... }\end{array}$ & $\alpha=, 729$ & 7 \\
\hline $\begin{array}{l}\text { Factor 2.- Valoración positiva y gusto por el trabajo en } \\
\text { equipo }\end{array}$ & $\alpha=, 699$ & 2 \\
\hline $\begin{array}{l}\text { Factor 3.- Atribuciones internas: los resultados y } \\
\text { calificaciones dependen del propio esfuerzo }\end{array}$ & $\alpha=, 438$ & 2 \\
\hline
\end{tabular}

Tabla 1. Fiabilidad de cada una de las dimensiones, ordenadas por porcentaje de varianza explicado.

\section{4.- Procedimiento}

En octubre, recién iniciado el curso, los estudiantes seleccionados contestaron este cuestionario junto a otros utilizados en la investigación.

En este primer pase de instrumentos a los alumnos se les pidió que contestasen los ítems del cuestionario pensando en su modo de afrontar el aprendizaje en el último curso del bachillerato, previo a su acceso a la universidad. Ello tenía su lógica porque se iba a analizar su evolución tanto en calificaciones como en procesos de aprendizaje desde la entrada en la universidad hasta el final de su segundo año en ella. Apenas tenían, en ese momento, experiencia en la universidad. Además, la primera toma de calificaciones realizada era la de la Prueba de Acceso a la Universidad (ésta fue la calificación

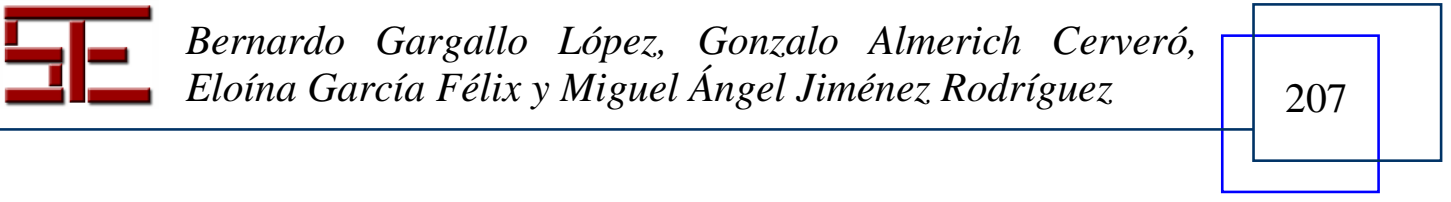




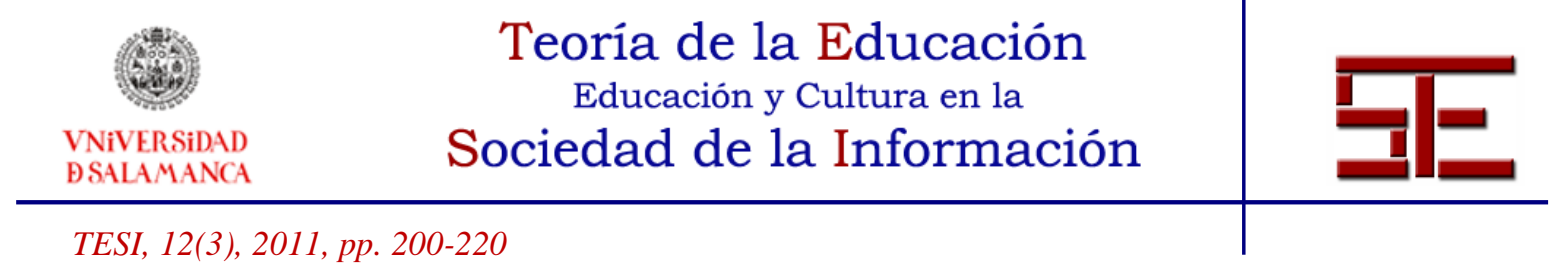

utilizada como medida del rendimiento para la clasificación de los alumnos en excelentes y medios; a lo largo de sus dos primeros años se irían recogiendo las calificaciones de las diferentes convocatorias).

Se utilizó para responder el cuestionario la plataforma de e-learning de la Universidad Politécnica de Valencia (PoliformaT) (https://poliformat.upv.es/portal), una plataforma muy versátil que permite alojar en ella instrumentos de evaluación y remitir las respuestas por la misma vía.

\section{4.- RESULTADOS}

Queríamos analizar las posibles diferencias existentes en actitudes ante el aprendizaje entre estudiantes excelentes y medios y también la validez predictiva de las mismas sobre el rendimiento académico.

\section{1.- Análisis de diferencias:}

Para analizar las posibles diferencias existentes entre estudiantes excelentes y medios realizamos dos aproximaciones diferentes: univariada y multivariada. La primera de ellas, univariada, se llevó a cabo mediante pruebas t de diferencia de significación de medias en los casos en que la distribución era normal y mediante la prueba no paramétrica U de Mann-Whitney en los casos en los que la distribución no lo era. La segunda de ellas, multivariada, se implementó mediante análisis discriminante para verificar las diferencias en cuanto al perfil de las variables consideradas.

\subsection{1.- Análisis de diferencias univariado:}

En la Tabla 2 figuran los resultados del análisis de diferencias univariado (*).

\begin{tabular}{|c|c|c|c|c|c|c|}
\hline $\begin{array}{l}\text { PUNTUACIONES DE ACTITUDES ANTE } \\
\text { EL APRENDIZAJE }\end{array}$ & $\begin{array}{l}\text { TIPOS DE } \\
\text { ALUMNOS }\end{array}$ & Media & $\mathbf{N}$ & $\begin{array}{l}\text { Desviación } \\
\text { típ. }\end{array}$ & gl & $\begin{array}{c}\text { Sig. } \\
\text { (bilateral) }\end{array}$ \\
\hline \multirow{2}{*}{$\begin{array}{l}\text { Factor 1.- Actitud positiva hacia el } \\
\text { aprendizaje profundo, crítico... }\end{array}$} & EXCELENTE & 3,8767 & 146 & ,55459 & 275 & \multirow{2}{*}{,002 } \\
\hline & MEDIO & 3,6750 & 131 &, 52952 & 273,927 & \\
\hline \multirow{2}{*}{$\begin{array}{l}\text { Factor 2.- Valoración positiva y gusto por el } \\
\text { trabajo en equipo }\end{array}$} & EXCELENTE & 3,9452 & 146 & 73373 & 275 & \multirow{2}{*}{,389 } \\
\hline & MEDIO & 4,0229 & 131 & ,75157 & 270,233 & \\
\hline \multirow[t]{2}{*}{ Factor 3.- Atribuciones internas } & EXCELENTE & 4,2877 & 146 &, 57785 & 275 & \multirow{2}{*}{,359 } \\
\hline & MEDIO & 4,2023 & 131 & 62258 & 266,101 & \\
\hline \multirow[t]{2}{*}{ Puntuación global } & EXCELENTE & 3,9639 & 146 & ,43833 & 275 & \multirow{2}{*}{,012 } \\
\hline & MEDIO & 3,8293 & 131 & ,44679 & 270,572 & \\
\hline
\end{tabular}

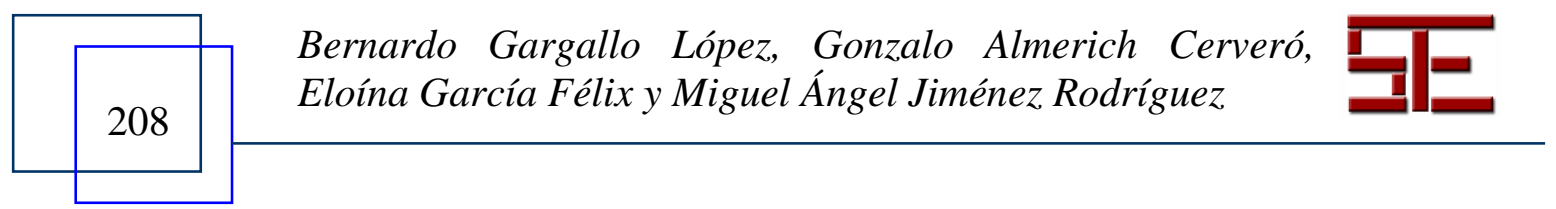




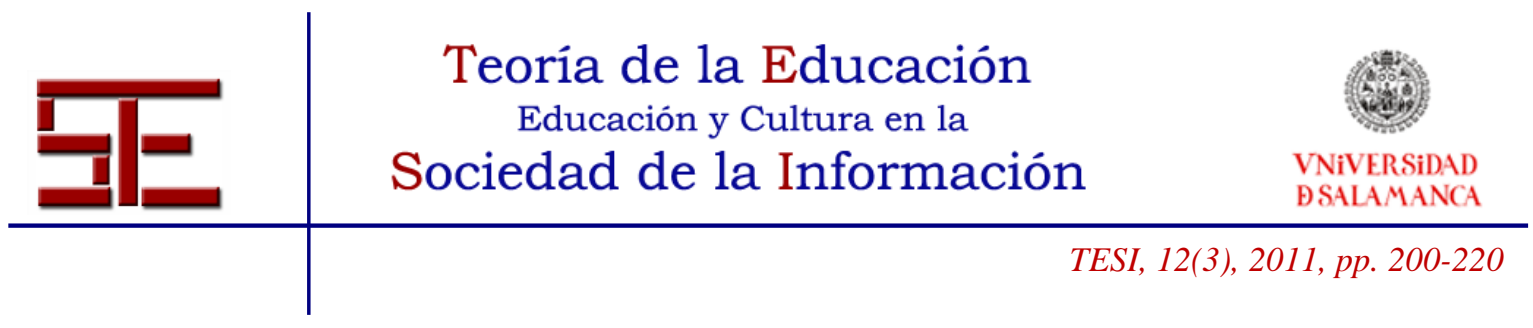

(*) Se han tomado los resultados de la significación de las diferencias eligiendo los que correspondían: en los casos en que la distribución no era normal se han utilizado los de la prueba no paramétrica U de Mann-Whitney; en los casos en que la distribución era normal se han tomado los de prueba la t de igualdad de medias para muestras independientes, atendiendo primero al cumplimiento o no de la igualdad de varianzas, contrastada mediante la $\mathrm{F}$ de Levenne.

Tabla 2. Análisis de diferencia de significación de medias en actitudes ante el aprendizaje entre estudiantes excelentes y medios en el primer pase.

Se presentan los resultados ordenados de la siguiente manera: en primer lugar las diferencias en las puntuaciones de los tres factores de estrategias (Factor 1, 2 y 3 ) y en segundo lugar las puntuaciones globales de actitudes.

Los resultados mostraron diferencias significativas en la puntuación total de actitudes $(\mathrm{p}<.05)$ y en la del primer factor $(\mathrm{p}<.01)$, que favorecían a los estudiantes excelentes, que disponían de mejores actitudes. No encontramos diferencias significativas en el segundo y tercer factor de la escala, siendo el segundo ligeramente superior en los medios y el tercero en los excelentes. Hay que tener en cuenta que el primer factor es el más potente de los tres en la validación del instrumento, y se refiere a la actitud positiva hacia el aprendizaje profundo, crítico, con comprensión, relacionando los contenidos; implica disposición al aprendizaje activo: a ampliar la información, a extraer consecuencia y supone gusto por el estudio en profundidad.

\subsection{2.-Análisis de diferencias multivariado:}

Para el análisis multivariado hemos utilizado análisis discriminante. El análisis discriminante es el procedimiento adecuado si se quiere profundizar en la interpretación de las diferencias que detectan el análisis univariado y multivariado. Las situaciones valoradas se basan en un modelo MANOVA simple, con una variable independiente de tipo entre (tipo de estudiantes en este caso) y múltiples variables dependientes. El análisis discriminante, en principio, es uno de los procedimientos más adecuados para identificar las relaciones causales potenciales respecto a las variables diferenciales y ofrecer una interpretación adecuada de las mismas. Este procedimiento permite no solamente verificar las diferencias entre los perfiles multivariados de los diferentes grupos que se comparan sino, también, establecer dimensiones que sintetizan estas diferencias. Estas dimensiones, similares a los factores del análisis factorial, simplifican la situación a un menor número de indicadores -la reducen-, y adquieren sentido en base a las relaciones que presentan con las variables dependientes originales. Asimismo, el análisis discriminante es útil para situaciones en las que se desea construir un modelo de

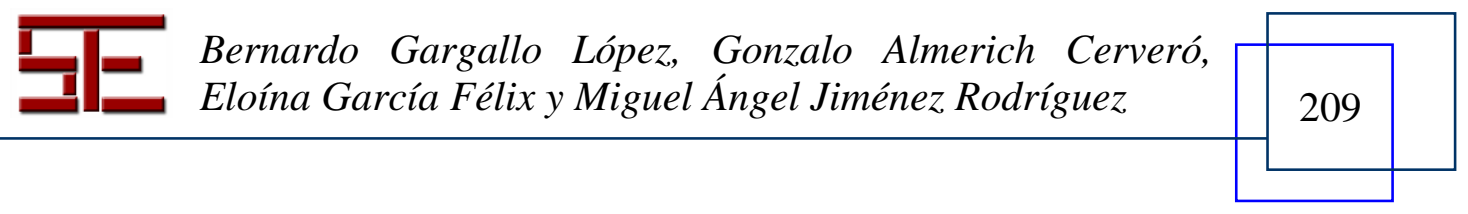




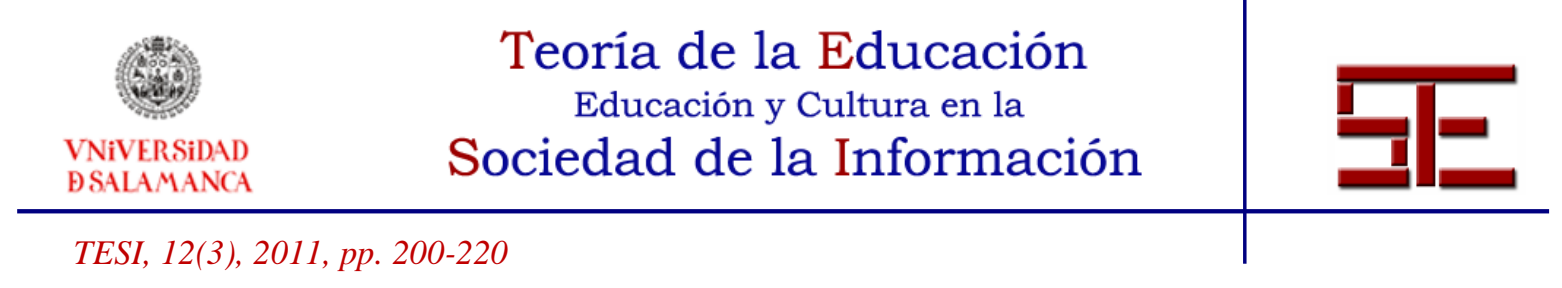

pronóstico de pertenencia al grupo basándose en las características observadas para cada caso. El procedimiento genera una función discriminante (o, para más de dos grupos, un conjunto de funciones discriminantes) basándose en las combinaciones lineales de las variables predictoras que proporcionan la mayor discriminación entre los grupos (Hair, Anderson, Tatham y Black, 1999).

El método seguido ha sido identificar a través de pruebas de significación univariadas y del correspondiente modelo multivariado -utilizando el procedimiento de paso-a-paso-, las variables que establecen diferencias significativas entre los grupos y llevar a cabo la solución completa del discriminante basada únicamente en dichas variables. De esta manera se ha utilizado el procedimiento según una aproximación de construcción de modelo, ya que no es ésta una situación en la que exista un modelo inicialmente propuesto, y se trata de indagar sistemáticamente para encontrar la mejor representación de las informaciones obtenidas. Con ello se ha pretendido evitar los riesgos que apuntan diferentes autores (Stevens, 1996) respecto a la utilización "automática" de procedimientos de análisis de datos, especialmente cuando los conocimientos asentados sobre una temática aconsejan incidir en una perspectiva exploratoria.

Los resultados obtenidos en la prueba $\mathrm{M}$ de Box (Tabla 3) nos indican que se cumple el supuesto de homogeneidad de la varianza en las poblaciones analizadas.

\begin{tabular}{|ll|r|}
\hline M de Box & & 5,020 \\
F & Aprox. &, 827 \\
& g11 & 6 \\
g12 & 530911,983 \\
& Sig. &, 549 \\
\hline \multicolumn{2}{|c|}{ Contrasta la hipótesis nula de que las matrices } \\
de covarianzas poblacionales son iguales.
\end{tabular}

Tabla 3. Prueba de Box.

Dado que el análisis discriminante se realiza sobre dos grupos poblacionales, obtenemos una única función discriminante. Dicha función presenta una correlación canónica moderada (.215) y un alto nivel de significación (.00) (Lambda de Wilks). En la tabla siguiente presentamos los coeficientes estandarizados y la matriz de estructura de la función canónica resultante.

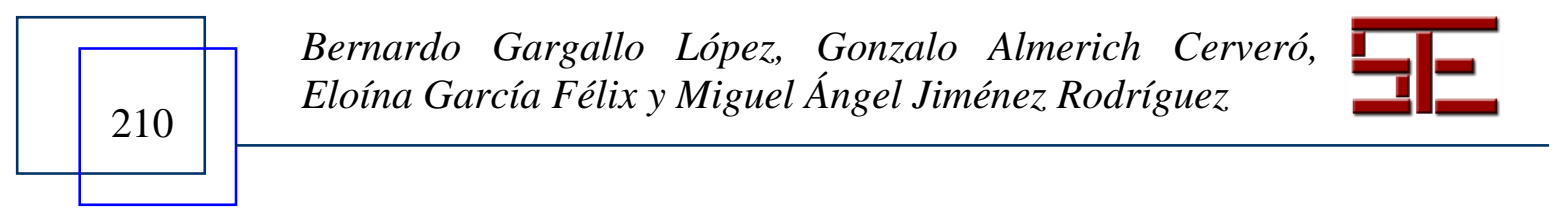




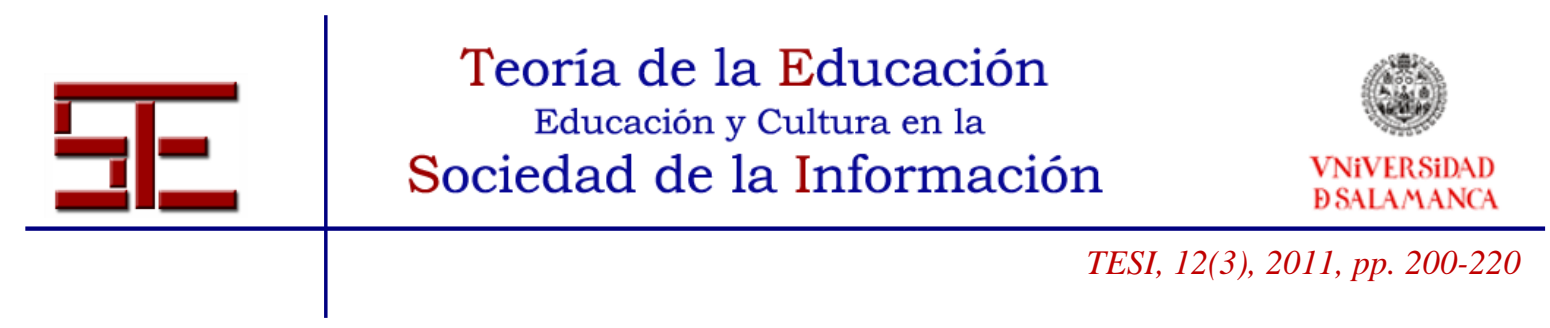

\begin{tabular}{|l|c|c|}
\hline & $\begin{array}{c}\text { Matriz de } \\
\text { estructura (a) }\end{array}$ & $\begin{array}{c}\text { Coeficientes estandarizados de las } \\
\text { funciones discriminantes canónicas }\end{array}$ \\
\hline Factor 1 Actitudes &, 846 &, 961 \\
Factor 2 Actitudes &,- 238 &,- 517 \\
Factor 3 Actitudes &, 324 &, 197 \\
\hline
\end{tabular}

(a) Correlaciones intra-grupo combinadas entre las variables discriminantes y las funciones discriminantes canónicas tipificadas.

Variables ordenadas por el tamaño de la correlación con la función.

Tabla 4. Puntuaciones en la matriz de estructura y coeficientes estandarizados

La función discriminante obtenida presenta una configuración bipolar. En el polo positivo, las dos puntuaciones del Factor 1 y del Factor 3 realizan una importante aportación a la función: la puntuación del Factor 1 (con un valor de ,846 de matriz de estructura y de ,961 de coeficiente estandarizado) y la del Factor 3 (con un valor de ,324 de matriz de estructura y de, 197 de coeficiente estandarizado). En el polo negativo la puntuación del Factor 2 realiza también una aportación similar, aunque no tan importante como el Factor 1 (con un valor de -,238 de matriz de estructura y de - ,517 de coeficiente estandarizado).

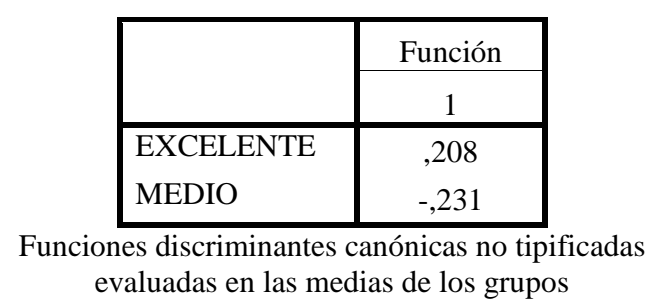

Tabla 5. Funciones en los centroides de los grupos

Por otro lado, los resultados de la función discriminante obtenida en los centroides de los grupos (ver Tabla 5) evidencian que los alumnos excelentes se sitúan en el polo positivo de la función $(, 208)$ y los medios en el negativo $(-, 231)$, lo que significa que los alumnos excelentes se orientan más hacia la Actitud positiva hacia el aprendizaje profundo, crítico, con comprensión, relacionando los contenidos... (Factor 1), y hacia las Atribuciones internas (Factor 3). Por el contrario, los alumnos medios se orientan hacia una Valoración positiva y gusto por el trabajo en equipo (Factor 2). No obstante, hay que tener presente que los valores obtenidos en las funciones de los centroides de ambos grupos indican que existe un cierto solapamiento entre ambos grupos.

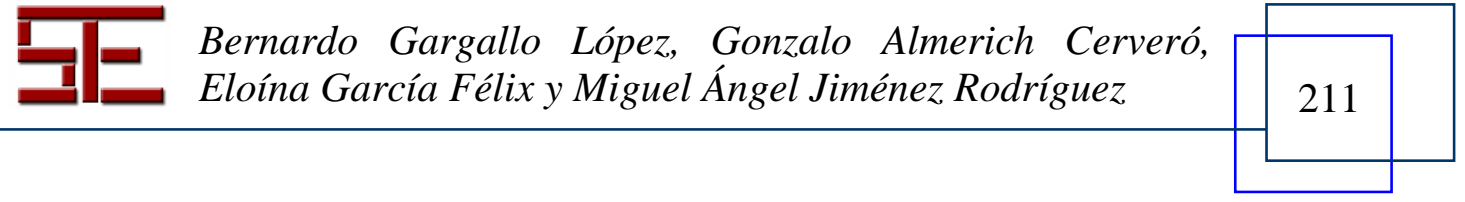




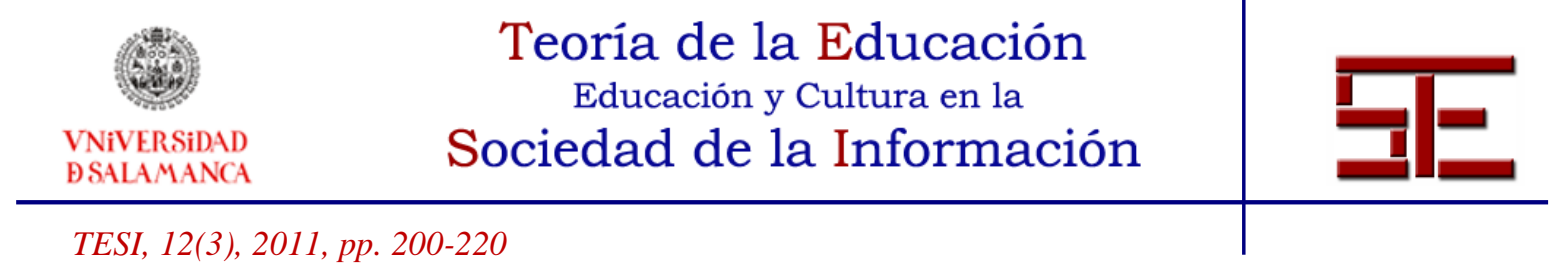

Estos resultados se constatan en la tabla presentada sobre los resultados de la clasificación de los alumnos (Tabla 6). En ella observamos que los alumnos excelentes alcanzan un porcentaje aceptable de clasificación correcta $(56,8 \%)$, mientras que los medios lo tienen un poco más alto $(58,0 \%)$. Hay un $58,8 \%$ bien clasificado de los casos agrupados originales y un 57,4\% de los casos agrupados mediante validación cruzada.

\begin{tabular}{|lll|r|r|r|}
\hline & & & \multicolumn{2}{|c|}{$\begin{array}{c}\text { Grupo de pertenencia } \\
\text { pronosticado }\end{array}$} \\
\cline { 4 - 5 } & & GMUESTRA2 & EXCELENTE & MEDIO & \multicolumn{1}{c|}{ Total } \\
\hline Original & Recuento & EXCELENTE & 84 & 62 & 146 \\
& & MEDIO & 52 & 79 & 131 \\
& & EXCELENTE & 57,5 & 42,5 & 100,0 \\
Validación cruzada(a) & \multirow{2}{*}{ Recuento } & EXCELENTE & 39,7 & 60,3 & 100,0 \\
& & MEDIO & 83 & 63 & 146 \\
& $\%$ & EXCELENTE & 55 & 76 & 131 \\
& & MEDIO & 56,8 & 43,2 & 100,0 \\
& & MEDIO & 42,0 & 58,0 & 100,0 \\
\hline
\end{tabular}

a La validación cruzada sólo se aplica a los casos del análisis. En la validación cruzada, cada caso se clasifica mediante las funciones derivadas a partir del resto de los casos.

b Clasificados correctamente el 58,8\% de los casos agrupados originales.

c Clasificados correctamente el $57,4 \%$ de los casos agrupados validados mediante validación cruzada.

Tabla 6. Resultados de la clasificación.

Estos resultados, complementarios de los aportados por el análisis univariado de diferencias, sirven para constatar, en todo caso, la mayor tendencia de los estudiantes excelentes a desarrollar Actitudes positivas hacia el aprendizaje profundo crítico y con comprensión y a preferir Atribuciones internas frente a los estudiantes medios, más proclives a desarrollar Actitudes positivas hacia el trabajo en equipo.

\section{2.- Resultados de validez predictiva:}

Efectuamos análisis de regresión lineal múltiple seleccionando como variables predictoras las puntuaciones medias de los tres factores actitudinales y como variable criterio la calificación de la Prueba de Acceso a la Universidad (que aparece como NOTAINGR en las tablas).

La regresión múltiple es un procedimiento idóneo para estudiar la relación entre una variable y múltiples variables. Se ocupa de la relación entre una variable dependiente o

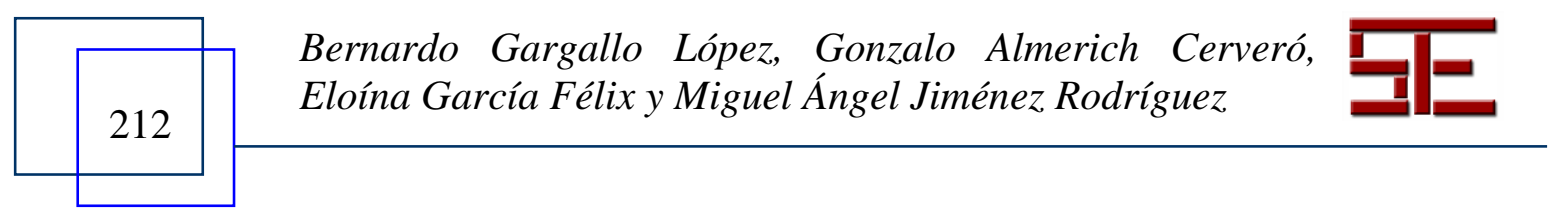




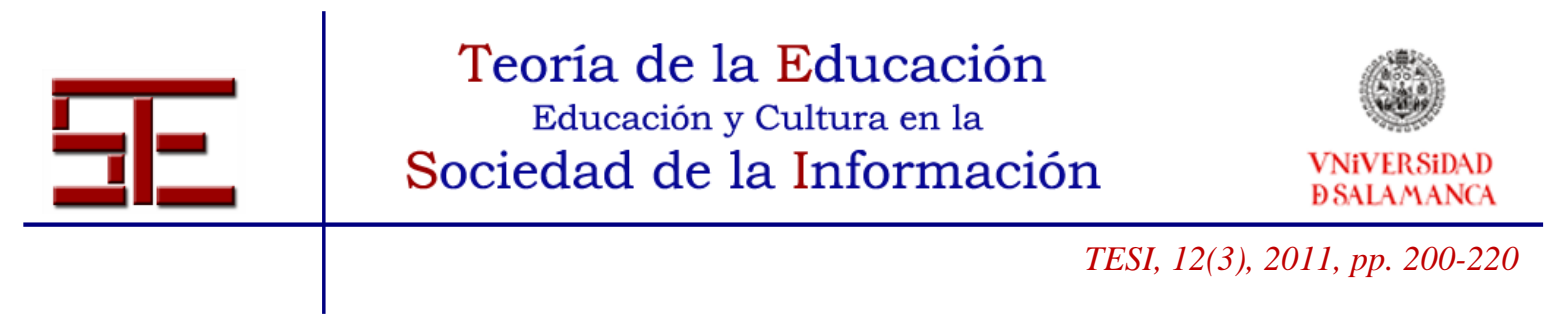

criterio $(Y)$ y un conjunto de variables independientes o predictores (las $X$ ). La regresión lineal múltiple estima los coeficientes de la ecuación lineal, con una o más variables independientes, que mejor prediga el valor de la variable dependiente.

Utilizamos el procedimiento "paso a paso", que permite determinar si una variable contribuye o no significativamente a la predicción. Si no fuera así, se elimina de la ecuación de regresión, con lo que el procedimiento permite obtener una ecuación de regresión en que todas las variables contribuyen significativamente a la predicción.

Utilizando este procedimiento los tres factores resultaron ser variables con contribución significativa en la predicción (Tabla 7). Encontramos un coeficiente "R" de correlación múltiple de 0,304 y un coeficiente de " $\mathrm{R}$ " de 0,92 , para el tercer modelo, que introduce las tres variables (los tres factores actitudinales), porque las tres contribuyen significativamente a la predicción, como se ha dicho. Ello supone que los tres predictores, actuando conjuntamente, explican aproximadamente el 9,2\% de la varianza de las calificaciones (Tabla 7).

\begin{tabular}{|c|c|c|c|c|c|c|c|c|c|}
\hline \multirow[b]{2}{*}{ Modelo } & \multirow[b]{2}{*}{$\mathrm{R}$} & \multirow[b]{2}{*}{$\begin{array}{c}\mathrm{R} \\
\text { cuadrado }\end{array}$} & \multirow[b]{2}{*}{$\begin{array}{l}\mathrm{R} \text { cuadrado } \\
\text { corregida }\end{array}$} & \multirow[b]{2}{*}{$\begin{array}{l}\text { Error típ. de la } \\
\text { estimación }\end{array}$} & \multicolumn{5}{|c|}{ Estadísticos de cambio } \\
\hline & & & & & $\begin{array}{c}\text { Cambio en } \mathrm{R} \\
\text { cuadrado }\end{array}$ & $\begin{array}{c}\text { Cambio en } \\
\mathrm{F}\end{array}$ & gl1 & $\mathrm{g} 12$ & $\begin{array}{l}\text { Sig. Cambio } \\
\text { en } \mathrm{F}\end{array}$ \\
\hline 1 & $236^{\mathrm{a}}$ &, 056 & 052 & ,99579 & 056 & 16,200 & 1 & 275 & 000 \\
\hline 2 & $272^{b}$ & 074 & 067 & ,98776 & 019 & 5,488 & 1 & 274 & 020 \\
\hline 3 & $304^{c}$ & ,092 & 082 & 97990 & 018 & 5,413 & & 273 & 021 \\
\hline
\end{tabular}

a. Variables predictoras: (Constante), Factor 1 de actitudes.

b. Variables predictoras: (Constante), Factor 1 de actitudes, Factor 2 de actitudes.

c. Variables predictoras: (Constante), Factor 1 de actitudes, Factor 2 de actitudes, Factor 3 de actitudes.

Tabla 7. Resultados del análisis de regresión múltiple. Resumen del modelo

La "F" de ANOVA para el conjunto de los tres predictores fue de 9,240, significativa al 0,001, lo que denota un buen nivel de predicción (Tabla 8).

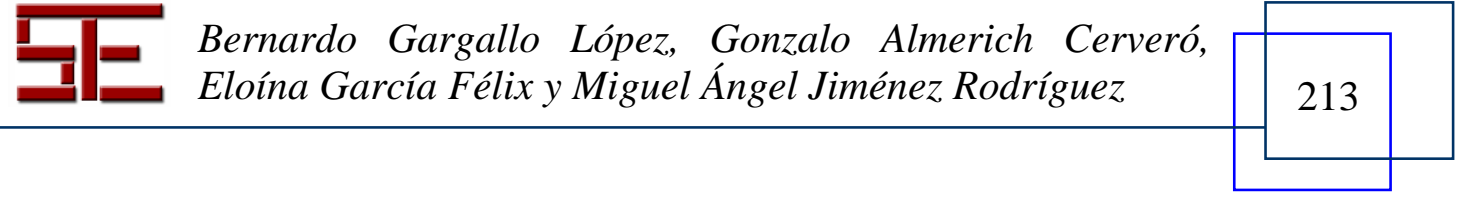




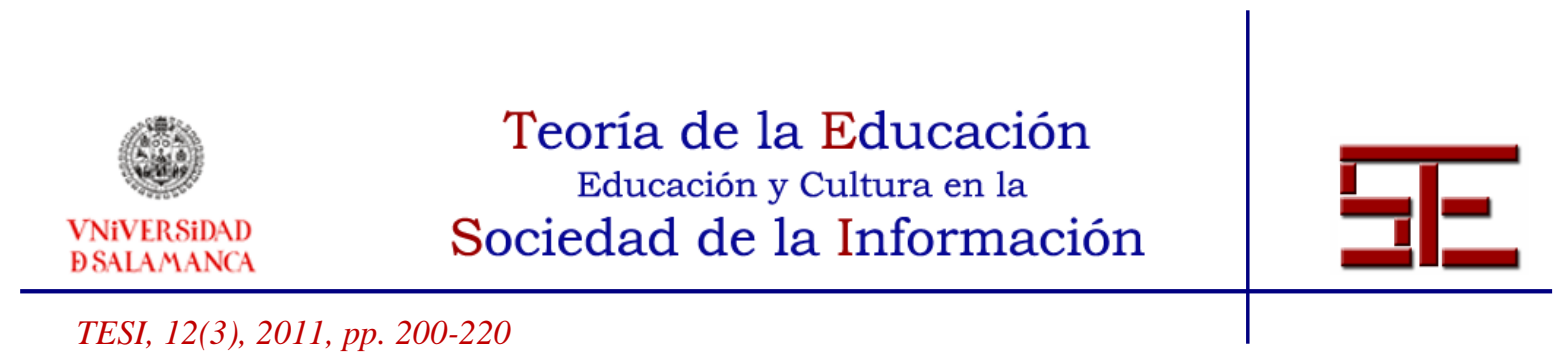

\begin{tabular}{|c|c|c|c|c|c|c|}
\hline & & $\begin{array}{c}\text { Suma de } \\
\text { cuadrados }\end{array}$ & gl & $\begin{array}{c}\text { Media } \\
\text { cuadrática }\end{array}$ & $\mathrm{F}$ & Sig. \\
\hline \multirow[t]{3}{*}{1} & Regresión & 16,064 & 1 & 16,064 & 16,200 & ,000(a) \\
\hline & Residual & 272,690 & 275 & ,992 & & \\
\hline & Total & 288,753 & 276 & & & \\
\hline \multirow[t]{3}{*}{2} & Regresión & 21,418 & 2 & 10,709 & 10,976 & ,000(b) \\
\hline & Residual & 267,335 & 274 & ,976 & & \\
\hline & Total & 288,753 & 276 & & & \\
\hline \multirow[t]{3}{*}{3} & Regresión & 26,616 & 3 & 8,872 & 9,240 &, $000(\mathrm{c})$ \\
\hline & Residual & 262,137 & 273 & ,960 & & \\
\hline & Total & 288,753 & 276 & & & \\
\hline \multicolumn{7}{|c|}{ a Variables predictoras: (Constante), Factor 1 de actitudes } \\
\hline \multicolumn{7}{|c|}{ Variables predictoras: (Constante), Factor 1 de actitudes, Factor 2 de actitudes } \\
\hline \multicolumn{7}{|c|}{ c Variables predictoras: (Constante), Factor 1 de actitudes, Factor 2 de actitudes, Factor 3 de actitudes } \\
\hline
\end{tabular}

Tabla 8. F de ANOVA

Es cierto que el primer factor (Valoración del aprendizaje profundo...) es el que aporta más peso a la predicción, ya que explica aproximadamente el 5,6\% de la varianza de las calificaciones (Tabla 7), con una alta significación ( $F$ de Anova de 16,200, p<,001 en el primer modelo). Aunque no es un valor predictivo demasiado alto, tampoco es un valor desdeñable (Beta de ,236 en el primer modelo y de ,251 en el tercero, que incluye los otros dos factores, significativas ambas al ,001 y correlación de ,236 en ambos modelos) (Tabla 9). El segundo factor (Valoración positiva y gusto por el trabajo en equipo) añade aproximadamente un 1,9\% a la explicación de la predicción, y el tercer factor (Atribuciones internas) un 1,8\% (Tabla 7). El signo de la predicción es positivo en el caso del primer y tercer factor, y negativo en el del segundo, lo que significa que tanto la Valoración del aprendizaje profundo como las Atribuciones internas predicen positivamente el rendimiento académico (a más peso de los factores mayor rendimiento académico) y, sin embargo, la Valoración positiva y el gusto por el trabajo en equipo lo hacen negativamente (a más peso menor rendimiento). Aunque los valores de los coeficientes beta y de las correlaciones son bajos en el segundo factor (Beta de -,140 significativa al ,05 y correlación de -,068 en el tercer modelo) y en el tercero (Beta de ,136, significativa al ,05 y correlación de ,172 en el tercer modelo) (Tabla 9) la aparente influencia negativa en las calificaciones de la Valoración y gusto por el trabajo en equipo merecería un trabajo posterior de análisis y constatación, con muestras más amplias.

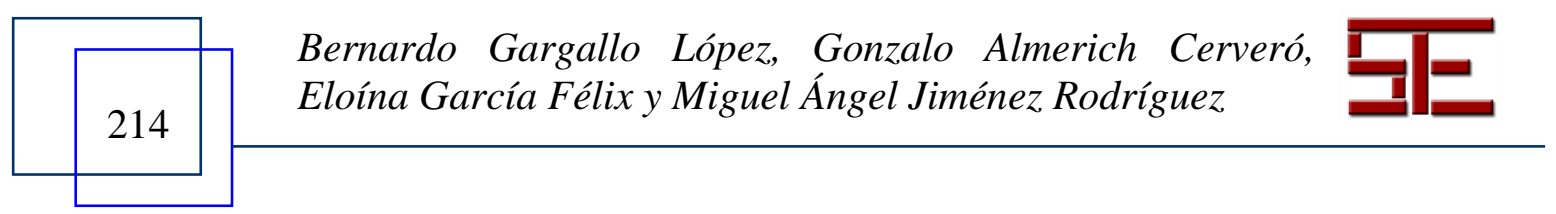




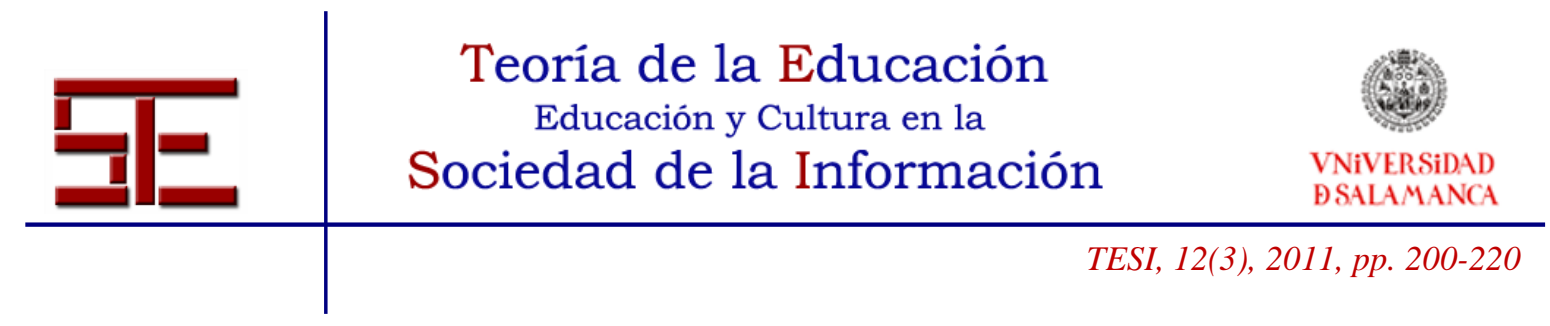

\begin{tabular}{|c|c|c|c|c|c|c|c|c|c|c|c|}
\hline \multicolumn{2}{|c|}{ Modelo } & \multicolumn{2}{|c|}{$\begin{array}{c}\text { Coeficientes } \\
\text { no } \\
\text { estandarizados }\end{array}$} & \multirow{2}{*}{$\begin{array}{c}\text { Coeficientes } \\
\text { estandarizados } \\
\\
\text { Beta } \\
\end{array}$} & \multirow{2}{*}{$\begin{array}{c}\mathrm{t} \\
\begin{array}{l}\text { Orden } \\
\text { cero }\end{array} \\
\end{array}$} & \multirow{2}{*}{$\begin{array}{c}\text { Sig. } \\
\text { Parcial } \\
\end{array}$} & \multicolumn{3}{|c|}{ Correlaciones } & \multicolumn{2}{|c|}{$\begin{array}{l}\text { Estadísticos de } \\
\text { colinealidad }\end{array}$} \\
\hline & & B & $\begin{array}{l}\text { Error } \\
\text { típ. }\end{array}$ & & & & Semiparcial & Tolerancia & FIV & B & Error típ. \\
\hline 1 & (Constante) & 6,381 & ,416 & & 15,358 & , 000 & & & & & \\
\hline \multirow{4}{*}{2} & $\begin{array}{l}\text { Dimensión } \\
\text { actitudes 1_I }\end{array}$ & ,438 & ,109 & ,236 & 4,025 & ,000 & ,236 & ,236 & ,236 & 1,000 & 1,000 \\
\hline & (Constante) & 6,892 & , 466 & & 14,779 & , 000 & & & & & \\
\hline & $\begin{array}{l}\text { Dimensión } \\
\text { actitudes 1_I }\end{array}$ &, 508 & ,112 & ,274 & 4,536 & ,000 & ,236 & ,264 & ,264 & ,929 & 1,077 \\
\hline & $\begin{array}{l}\text { Dimensión } \\
\text { actitudes 2_I }\end{array}$ &,- 195 & ,083 &,- 141 & $-2,343$ & ,020 &,- 068 &,- 140 &, 136 & ,929 & 1,077 \\
\hline \multirow[t]{4}{*}{3} & (Constante) & 6,062 & ,584 & & 10,376 & , 000 & & & & & \\
\hline & $\begin{array}{l}\text { Dimensión } \\
\text { actitudes 1_I }\end{array}$ & ,465 &, 113 & ,251 & 4,137 & ,000 & ,236 & ,243 & ,239 & ,905 & 1,105 \\
\hline & $\begin{array}{l}\text { Dimensión } \\
\text { actitudes 2_I }\end{array}$ &,- 194 & ,082 &,- 140 & $-2,348$ & ,020 &,- 068 &,- 141 & ,135 & ,929 & 1,077 \\
\hline & $\begin{array}{l}\text { Dimensión } \\
\text { actitudes 3_I }\end{array}$ & ,232 & , 100 & , 136 & 2,327 & ,021 & , 172 & ,139 & ,134 & ,973 & 1,028 \\
\hline
\end{tabular}

a Variable dependiente: NOTAINGR.

Tabla 9. Coeficientes.

\section{5.- CONCLUSIONES}

Los resultados obtenidos muestran que los estudiantes excelentes desarrollan mejores actitudes ante el aprendizaje que los estudiantes medios, tanto en la puntuación global de actitudes como en dos de los factores integrantes, lo que se ha corroborado mediante análisis de diferencias univariado y multivariado: muestran una Actitud más comprometida hacia el aprendizaje profundo, crítico y con comprensión (Factor 1) y desarrollan Atribuciones internas (Factor 3). Los estudiantes medios muestran una inclinación ligeramente superior a los excelentes en la Valoración positiva y el gusto por el trabajo en equipo (Factor 2).

Por otra parte, el análisis de regresión efectuado corroboró el poder predictivo de las actitudes sobre el rendimiento académico, en línea positiva en el caso de los Factores 1 y 3 y en línea negativa en el caso del Factor 2.

Queremos llamar la atención sobre el hecho de que precisamente los factores en que los alumnos excelentes presentan diferencias favorables frente a los medios son los que presentan incidencia positiva sobre el rendimiento.

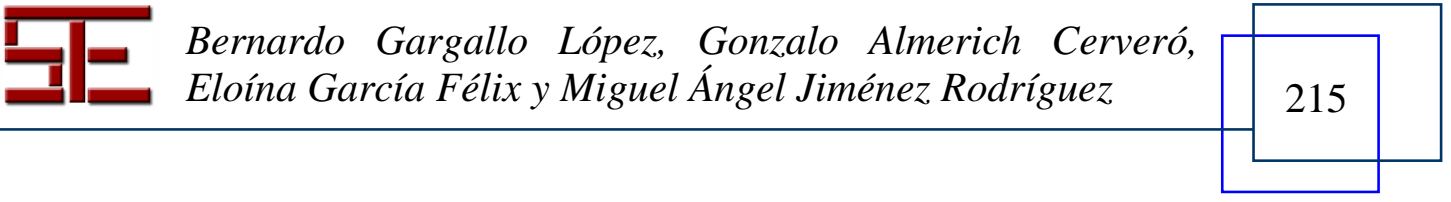




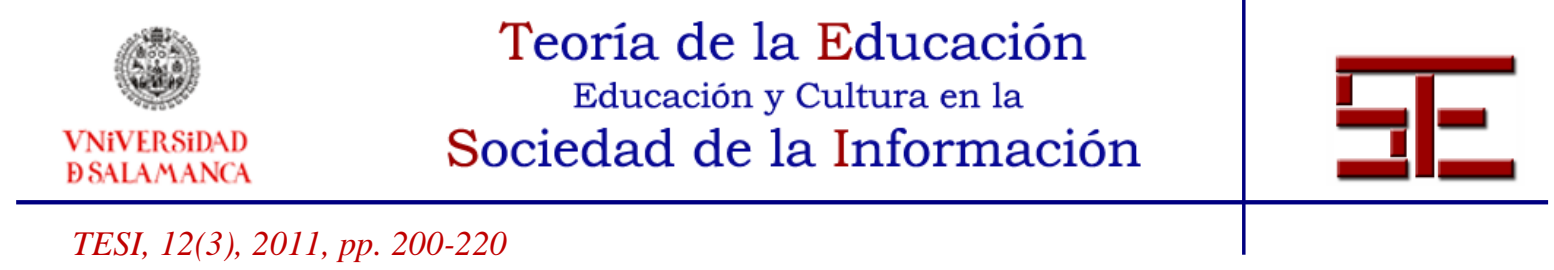

La excelencia tiene que ver, pues, con las actitudes ante el aprendizaje, y específicamente con la Actitud positiva hacia el aprendizaje profundo, crítico y con comprensión... y con las Atribuciones internas.

Estos resultados reafirman los provenientes de diversos estudios que confirman la influencia de las actitudes ante el aprendizaje en el rendimiento académico de los estudiantes (Gargallo, Pérez, Serra, Sánchez y Ros, 2007; Goolsby, 1988; Herrero, Nieto, Rodríguez y Sánchez, 1999; House y Prion, 1998).

La mayor asociación de los estudiantes medios con la Valoración positiva y el gusto por el trabajo en equipo y la incidencia negativa de este factor sobre el rendimiento académico merecería un estudio posterior, más profundo y con mayor muestra, como se ha referido antes. En todo caso, puede tener que ver con una mayor independencia de los estudiantes excelentes y con una cierta percepción de la no necesidad de trabajar con compañeros, porque pueden estimar que éstos no les aportan demasiado o los retrasan, lo que debería hacernos reflexionar a los profesores tanto sobre la necesidad de trabajar la actitud positiva hacia el trabajo en equipo también en los alumnos con mayor rendimiento, como sobre la necesidad de desarrollar en nuestras materias buenos procedimientos de trabajo en equipo (que hay que diseñar bien, entrenar, pautar, seguir en su ejecución, etc.; el buen trabajo en equipo se aprende y se puede enseñar y no basta con exigir a los alumnos que lo realicen sin supervisión para tener garantía de un trabajo de calidad).

La constatación de la incidencia de las actitudes sobre el rendimiento y la necesidad, por tanto, de desarrollar buenas actitudes, específicamente las recogidas en los Factores 1 y 3 , suponen un claro reto para los profesores universitarios, que debemos potenciarlas en los estudiantes, por una parte, porque éstos aprenderán más y mejor, y por otra porque así es mucho más probable que les vaya bien en los estudios.

Tenemos datos de nuestras investigaciones (Gargallo, 2006 y 2008) que prueban que la metodología de enseñanza y aprendizaje del profesor influye significativamente en el modo de trabajar del estudiante. En el caso de los actitudes, cuando los profesores suscriben planteamientos centrados en el aprendizaje y utilizan metodologías de enseñanza y evaluación adecuadas, los alumnos desarrollan mejores actitudes, al contrario de lo que ocurre cuando se suscriben planteamientos centrados en la enseñanza y cuando los profesores se centran en la metodología expositiva sin otras

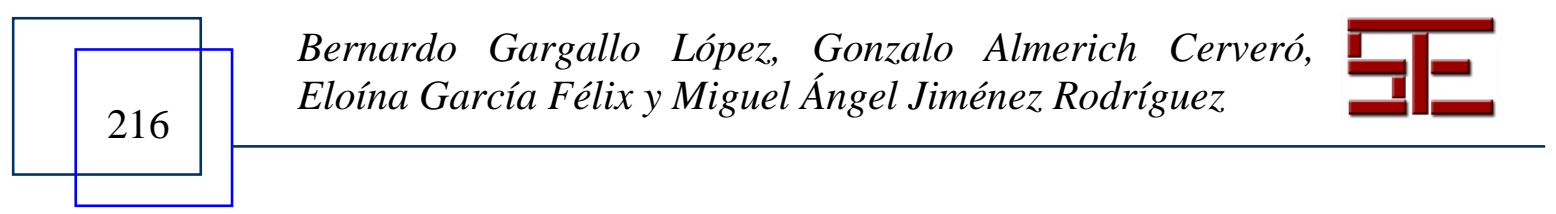




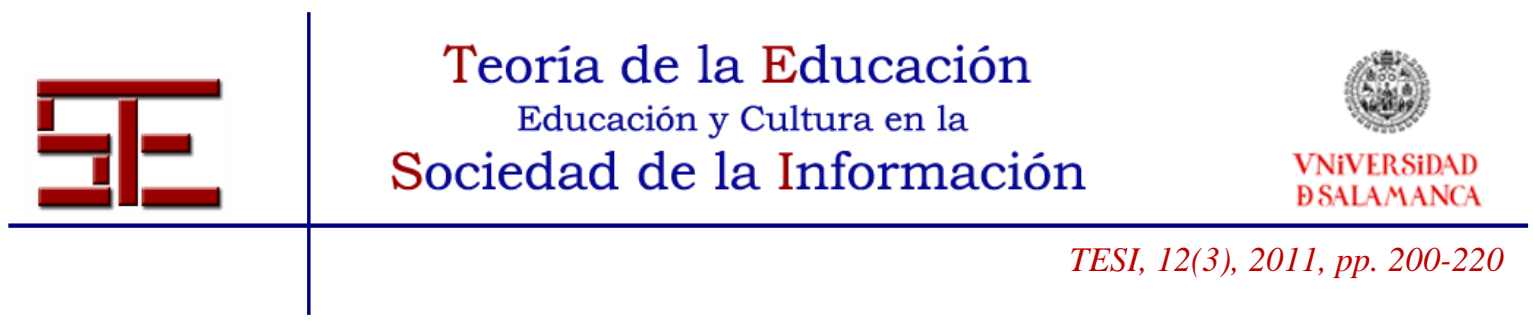

alternativas $\mathrm{y}$ en el examen final como método de evaluación, frente a otros procedimientos formativos.

Es cierto que en ello juegan muchas variables, con toda seguridad, como motivos, intereses, enfoques de aprendizaje, idiosincrasia personal, expectativas, experiencia previa, manejo de estrategias de aprendizaje, etc., todas ellas provenientes del estudiante, pero también lo es que los profesores podemos potenciar el desarrollo de actitudes positivas en nuestra actuación docente en la universidad promoviendo metas de alto nivel para los estudiantes que vayan más allá de la reproducción de los conocimientos, utilizando metodologías de enseñanza más adecuadas que la sola enseñanza expositiva: es verdad que la metodología expositiva de calidad es necesaria (siempre que las lecciones magistrales sean lecciones magistrales, no pura lectura de apuntes), pero puede ser complementada con otros procedimientos: la resolución de problemas, el estudio de casos, el diseño de proyectos, el planteamiento de preguntas, la discusión y negociación en el aula, etc. son instrumentos adecuados. Los profesores debemos fomentar el pensamiento crítico y el compromiso de los estudiantes y estos métodos ayudan a ello. Ayuda también arbitrar procedimientos de evaluación más exigentes que vayan más allá de la pura reiteración de lo aprendido, con orientación formativa, que devuelva feed-back a los estudiantes, etc. Éste es, sin duda, un reto apasionante en un nuevo escenario promovido por la convergencia europea en el que ya estamos.

\section{BIBLIOGRAFÍA}

Akey, T. (2006). School context, student attitudes and behaviour, and academic achievement: An exploratory analysis. Informe de investigación. Extraído el 20 de julio, 2011, de http://www.mdrc.org/publications/419/full.pdf.

Ajzen, I. \& Madden, T. J. (1986). Predicting goal-directed behavior:attitudes, intentions and perceived behavioral control. Journal of Experimental Social Psychology, $23,453-47$.

Amezcua, J. A. y Fernández, E. (2000). La influencia del autoconcepto en el rendimiento académico. Iberpsicología, 5.1. Extraído el 10 de octubre, 2007, de http://www.fedap.es/IberPsicologia/iberpsi5-1/amezcua/amezcua.htm.

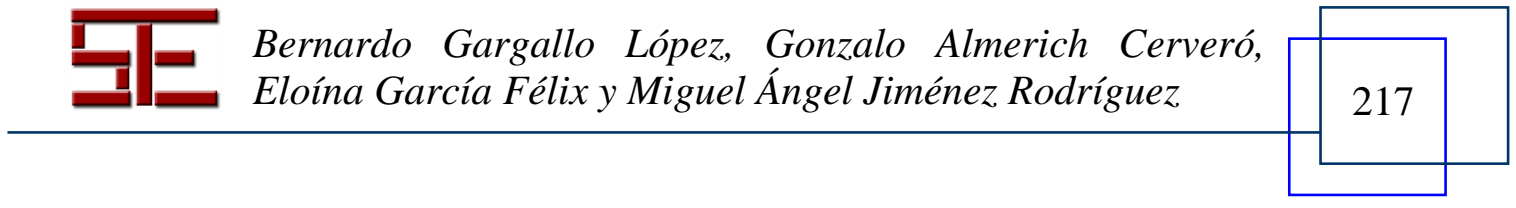




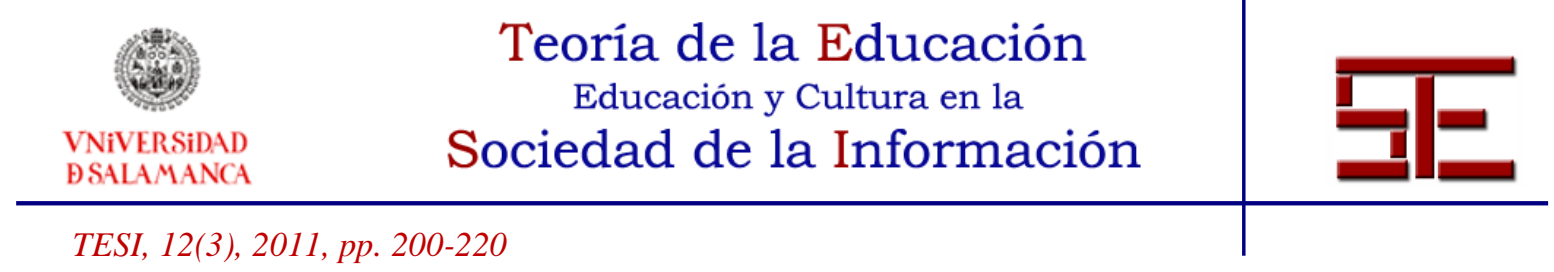

De Miguel, M. y Arias, J. M. (1999). La evaluación del rendimiento inmediato en la enseñanza universitaria. Revista de Educación, 320, 353-377.

Deronck, N. G. (2007). The impact of family structure and involvement on the college enrollment of potential first-generation college students. Dissertation Abstracts International Section A: Humanities and Social Sciences, vol. 68 (5-A), 1827.

Escámez, J., García, R., Pérez, C. y Llopis, A. (2007). El aprendizaje de actitudes y valores. Teoría y práctica. Barcelona: Octaedro-OEI.

Escámez, J. y Ortega, P. (1986). La enseñanza de actitudes y valores. Valencia: Nau Libres.

Fishbein, M. and Ajzen, I. (1980). Understanding attitude and predicting social behavior. Nueva Jersey: Prentice Hall.

Fore, Ch. L. (1998). Factors influencing academic achievement among Native American college sudents. Dissertation Abstracts International: Section B: The Sciences and Engineering, vol. 58 (8-B), 4522.

García, R. y Sales, A. (1997). Programas de educación intercultural. Bilbao: Desclée de Brower.

Gargallo, B., Pérez, C., Fernández, A. y Jiménez, M.A. (2007). La evaluación de las actitudes ante el aprendizaje de los estudiantes universitarios. El Cuestionario CEVAPU. Teoría de la Educación. Educación y Cultura en la Sociedad de la Información, 8 (2), 238-258. Extraído el 17 de julio, 2011 de http://www.usal.es/ teoriaeducacion/rev_numero_08_02/n8_02_gargallo_perez_ fernandez_jimenez.pdf.

Gargallo, B., Pérez, C., Serra, B., Sánchez, F. y Ros, C. (2007). Actitudes ante el aprendizaje y rendimiento académico en estudiantes universitarios. Revista Iberoamericana de Educación, 42, 1-25.

Goolsby, Ch. B. (1988). Factors affecting mathematics achievement in high risk college students. Research \& Teaching in Developmental Education, 4(2), 18-27.

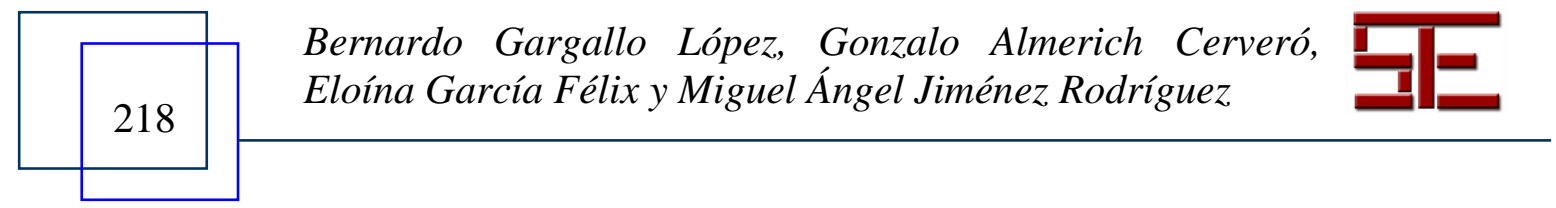




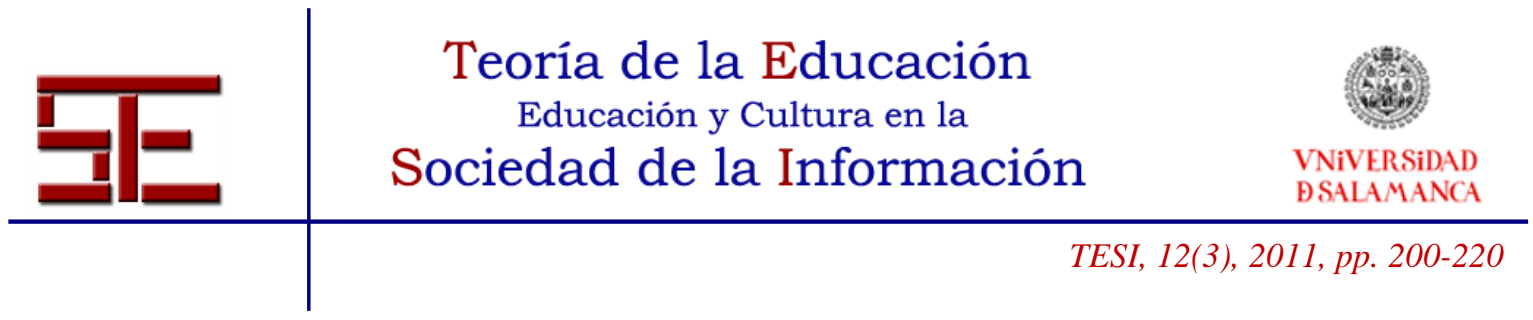

Hair, J.F., Anderson, R.E., Tatham, R.L. y Black, W.C. (1999). Análisis Multivariante. ( $5^{\text {a }}$ ed.). Madrid: Prentice Hall.

Herrero, $M^{a}$.E., Nieto, S., Rodríguez, $M^{a} . J$. y Sánchez, $M^{a}$.C. (1999). Factores implicados en el rendimiento académico de los alumnos de la Universidad de Salamanca. Revista de Investigación Educativa, 17 (2), 413-421.

House, J. D. \& Prion, S.K. (1998). Student attitudes and academic background as predictors of achievement in college English. International Journal of Instructional Media, 25 (1), 29-42.

Meléndez, D. (2007). Un ejercicio de evaluación de las trayectorias escolares en la Universidad de Guadalajara. Comunicación presentada en el $6^{\circ}$ Congreso Internacional Retos y Expectativas de la Universidad "El papel de la universidad en la transformación de la sociedad". Extraído el 21 de diciembre, 2011 , http://www.congresoretosyexpectativas.udg.mx/Congreso\%206/Eje\%202/Ponen cia_111.pdf.

Pritchard, M. E., Wilson, G. S. y Yamnitz, B. (2007). What predicts adjustment among college students? A longitudinal panel study. Journal of American College Health, 56 (1), 15-21.

Quiles, Ma . N. (1993). Actitudes hacia las matemáticas y rendimiento escolar. CL\&E. Comunicación, Lenguaje y Educación, 18, 115-125.

Ramírez, Mª J. (2005). Actitudes hacia las matemáticas y rendimiento académico entre estudiantes de octavo básico. Estudios pedagógicos, 31 (1), 97-112.

Rayle, A. D. y Chung, K. (2007). Revisiting first-year college students' mattering: Social support, academic stress, and the mattering experience, Journal of College Students Retention: Research, Theory and Practice, 9: 1, 21-37.

Rokeach, M. (1970). Beliefs, Acttitudes and values. San Francisco: Jossey-Bass.

- (1979). Some unresolved issues in theories of beliefs, attitudes and values. Act. Nebraska symposium on motivation.

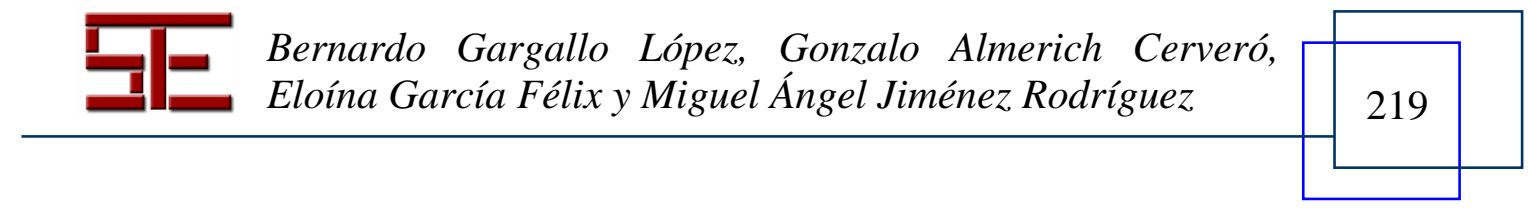


Stevens, J. (1996). Applied Multivariate Statistics for the Social Sciences. (3 ${ }^{\mathrm{a}}$. Ed.) Mahwah: NJ.LEA.

Strayhorn, T. L. (2006). Factors Influencing the Academic Achievement of FirstGeneration College Students. NASPA Journal, 43 (4), 82-111.

Vander Zanden, J. W. (1989). Manual de psicología social. Buenos Aires: Paidós.

Para citar el presente artículo puede utilizar la siguiente referencia:

Gargallo López, B., Almerich Cerveró, G., García Félix, E. y Jiménez Rodríguez, M. A. (2011). Actitudes ante el aprendizaje en estudiantes universitarios excelentes y en estudiantes medios. Revista Teoría de la Educación: Educación y Cultura en la Sociedad de la Información. 12(3), 200-220 [Fecha de consulta: dd/mm/aaaa]. http://campus.usal.es/ revistas_trabajo/index.php/revistatesi/article/view/8489/8582

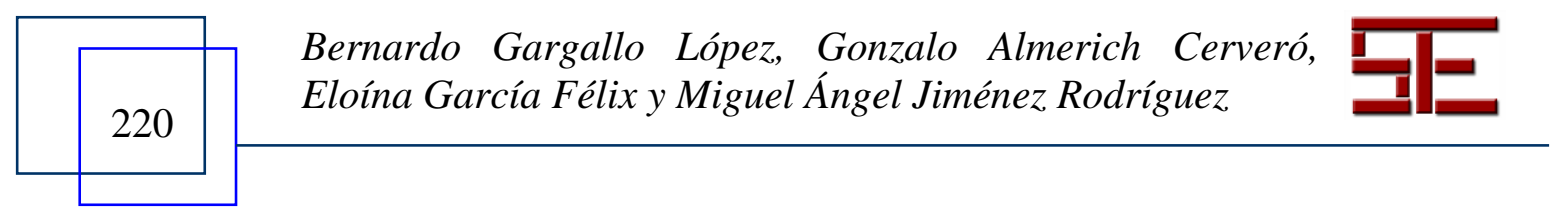

